\title{
Pratiques touristiques et apprentissages
}

\section{Gilles Brougère}

\section{OpenEdition}

\section{Journals}

Édition électronique

URL : http://journals.openedition.org/tourisme/415

DOI : 10.4000/tourisme.415

ISSN : 2492-7503

\section{Éditeur}

Éditions touristiques européennes

\section{Édition imprimée}

Date de publication : 1 juin 2012

Pagination : 62-75

ISSN : 2109-5671

\section{Référence électronique}

Gilles Brougère, "Pratiques touristiques et apprentissages », Mondes du Tourisme [En ligne], 5 | 2012, mis en ligne le 30 septembre 2015, consulté le 19 avril 2019. URL : http://journals.openedition.org/ tourisme/415; DOI : 10.4000/tourisme.415

\section{(c) (i) (3)}

Mondes du tourisme est mis à disposition selon les termes de la licence Creative Commons Attribution - Pas d'Utilisation Commerciale - Pas de Modification 4.0 International. 


\title{
Pratiques touristiques et apprentissages
}

\author{
GILLES BROUGÈRE
}

[brougere@univ-paris|3.fr]

Professeur de sciences de l'éducation

Directeur d'Experice (Centre de recherche interuniversitaire Expérience, ressources culturelles, éducation), université Paris 13

Résumé. Si nombre de disciplines contribuent aux études sur le tourisme, celui-ci n'est pas un objet des sciences de l'éducation et, plus largement, l'étude des relations entre tourisme et éducation ou la question de savoir si les pratiques touristiques peuvent être des espaces d'apprentissage sont peu présentes dans la littérature. Certes, de la référence au Grand Tour à celle du tourisme culturel, en passant par la question du nécessaire apprentissage du tourisme comme pratique spécifique, on trouve des évocations de l'éducation ou des apprentissages, mais peu développées et le plus souvent en dehors de toute recherche empirique. II nous semble qu'il manque une théorie, des concepts permettant de penser une telle relation. Nous partons des théories de "l'apprentissage situé" pour les confronter et les adapter à des données empiriques (observations et entretiens) issues d'une recherche sur le tourisme social. Sur cette base, nous proposerons une architecture conceptuelle autour des notions de participation guidée et d'exploration guidée permettant de penser les effets d'apprentissage de pratiques touristiques.

Abstract. Many academic disciplines contribute to the tourism studies, but it is not the case for educational sciences. More generally the issue of the relationship between education and tourism, or those of the tourism practice as learning place has no room in the scientific literature. The reference to the Grand Tour, to cultural tourism, or to the question of the necessity to learn the tourism practice as all other practices, involve some remarks about learning in tourism context, but undeveloped and with few empirical data. It lacks of theories, concepts to think about such relationship. The article uses the theories of situated learning to confront and to adapt its to empirical data (observations and interviews) from a research about social tourism. We propose a conceptual architecture with the notions of guided participation and guided exploration, which are useful to think about the learning effects of tourism practices. 


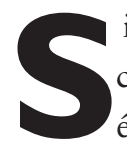
i beaucoup de disciplines contribuent aujourd'hui aux études sur le tourisme, il semble bien que la contribution des sciences de l'éducation soit proche de zéro, si l'on ne tient pas compte de ce qui concerne non le tourisme lui-même mais son enseignement dans des écoles ou universités. Que l'on considère cette discipline comme spécifique ou simplement comme l'association de diverses disciplines pour étudier l'éducation au sens le plus large (ce qu'il conviendrait d'appeler symétriquement les études sur l'éducation), le tourisme ne fait pas partie de ses objets, lors même qu'elle s'intéresse à d'autres situations que l'école ou l'éducation formelle, par exemple au travail. Tout se passe comme si les activités frivoles, de récréation, de loisir avaient bien des difficultés à trouver place au sein de cette discipline.

Du côté des études du tourisme malgré la relative évidence d'une relation avec des questions d'apprentissage ou d'éducation, depuis le Grand Tour jusqu'au tourisme culturel, en passant par certains objectifs du tourisme social ou la place des apprentissages corporels, les travaux ou réflexions portant sur les relations entre tourisme et apprentissage ou éducation sont rares. Il s'agit le plus souvent d'analyses sporadiques qui ne conduisent pas, malgré leur intérêt, à un développement de recherches centrées sur cette question. Elles semblent manquer de concepts permettant de construire une relation forte entre apprentissage et tourisme.
Il nous semble que cette situation appelle une réflexion sur la façon dont on peut penser la question de l'apprentissage au sein des pratiques touristiques, ou plutôt, formulation que nous préférons, les pratiques touristiques comme espace d'apprentissage. Cela implique de disposer de concepts qui, pris dans diverses théories de l'éducation ou de l'apprentissage, et moyennant des modifications, peuvent s'appliquer au tourisme.

Le but de cet article est de proposer un cadre conceptuel issu des théories forgées pour penser certaines situations éducatives pouvant permettre de théoriser la dimension éducative des pratiques touristiques. Mais notre façon de faire de la recherche nous interdit de déployer cet article uniquement au niveau théorique ou conceptuel.

En effet, nous pensons que, si la construction théorique n'est pas toujours et seulement issue de l'empirie selon le modèle de la théorisation ancrée (Glaser et Strauss, 20 I0), elle ne peut pour autant se développer sans confrontation avec un terrain, des données. Nous nous inscrivons ici dans le modèle de la "théorie adaptative” proposée par Derek Layder (1998), qui consiste à s'appuyer sur des éléments théoriques pour les adapter en permanence en fonction des données empiriques. Si la théorie n'est pas uniquement produite par le terrain, elle n'est pas pour autant une construction decontextualisée que les données devraient seulement valider. Ou, pour le dire autrement, la théorie que nous esquissons et que nous soumettons à la critique des spécialistes du tourisme est tout autant issue de théories antérieures que de confrontations avec un terrain de recherche que nous présenterons. Il s'agit de faire des allers et retours entre théories disponibles et données de terrain.

Notre recherche sur le tourisme s'intègre dans un programme de recherche plus large sur les pratiques sociales comme espace d'apprentissage : qu'apprend-on et comment dans le cadre de pratiques qui n'ont pas pour objectif l'apprentissage ? Ce que la littérature internationale nomme "apprentissage informel" a été l'objet d'explorations diverses (Brougère et Bézille, 2007 ; Brougère et Ulmann, 2009), en particulier par les tenants de l'apprentissage situé (Lave et Wenger, 1991 ; Wenger, 2005). Nous disposons ainsi, sinon d'une théorie, tout au moins d'un ensemble théorique et conceptuel relatif à l'apprentissage en situation informelle. Cette recherche s'enracine dans l'analyse de situations de travail ou d'autres situations qui semblent en être proches. Elle met l'accent sur l'idée que l'apprentissage n'est pas nécessairement conscient, ou, s'il l'est, qu'il peut être fortuit ou, s'il n'est pas fortuit, qu'il peut rester informel car autodirigé, organisé et régulé par la personne elle-même (Schugurensky, 2007). On peut ainsi apprendre non seulement sans école, mais également sans enseignant ni pédagogie. Cet apprentissage ${ }^{(1)}$ renvoie à une logique de participation : c'est en participant que l'on apprend, et la motivation est celle d'être 
membre d'un groupe, d'où l'idée de communautés de pratique caractérisées par un engagement mutuel, une entreprise commune et un répertoire partagé (Wenger, 2005). Enfin cette participation a pu être analysée par Billett (2004) comme une relation étroite entre engagement de la personne dans la situation et affordance $^{(2)}$ de la situation (ce que celleci offre à celui qui y participe). Les modalités peuvent être diverses. Ce corps de théorie, adapté à des situations de travail, semble s'appliquer au jeu de façon générale (Brougère, 2005), et à des activités plus spécifiques comme le jeu en ligne (Berry, 2009). Peut-il s'appliquer au tourisme de façon à rendre visible des apprentissages qui, par définition, sont implicites et invisibles?

\section{QUELQUES PISTES POUR PENSER LES LIENS ENTRE}

\section{TOURISME ET ÉDUCATION}

Certains (Cohen, 1985 ; Mitchell, 1998) ont pensé trouver dans les proximités entre jeu et tourisme de quoi justifier la dimension éducative de cette activité. Mais la critique de l'évidence de la relation entre jeu et éducation (Brougère, 1995, 2005 ; Power, 2000 ; Sutton-Smith, 1997) ne permet pas de fonder, sinon de façon mythique, la relation sur cette base, d'autant plus que si le jeu est une des dimensions centrales du tourisme, il ne se confond pas avec le tourisme, qui implique également repos, sociabilité, divertissement, découverte (Équipe Mit, 20II), activités qui ne peuvent être confondues avec le jeu.
Au-delà de cette tentative d'utiliser le paradigme ludique, il y a peu de recherches qui mettent en évidence la question éducative dans les pratiques touristiques. C'est relativement paradoxal dans la mesure où chacun relève l'origine du terme, sinon de la pratique contemporaine, dans le Grand Tour, voyage initiatique en Europe, permettant de découvrir les États européens et de s'exercer aux pratiques sociales de leur classe, effectué par les jeunes nobles du XVI ${ }^{\mathrm{e}}$ au XVIII' siècle (Towner, 1985 ; Boutier, 2004). Cependant, comme bien souvent, l'origine étymologique doit être prise avec distance. Si le Grand Tour est bien affaire d'éducation, s'il s'agit bien de voyager, nous sommes très loin des pratiques touristiques contemporaines : "Le Grand Tour constitue la mise à l'épreuve d'une éducation nobiliaire dont l'économie, commune aux principales nations européennes, s'est constituée à la Renaissance; elle articule les disciplines militaires (équitation, maniement des armes, mathématiques) et les arts mondains (danse, musique); l'apprentissage politique (histoire, géographie, droit) et la connaissance des moyens de communication (langues étrangères)" (Boutier, 2004, pp. 8-9). Il s'agit d'un voyage d'éducation sans doute plus proche, si l'on veut le traduire dans le monde contemporain, d'un stage de fin d'études que du tourisme de loisir qui nous intéresse ici. Que le voyage dans ses formes les plus nobles, les plus valorisées soit éducatif et forme tout particulièrement la jeunesse, cela est relativement reconnu et inté- gré à nombre de curriculums contemporains, en particulier au niveau européen avec le programme Erasmus. Reste la question de savoir si "l'idiot du voyage" (Urbain, 2002) apprend également. Il s'agit bien de s'intéresser aux pratiques les plus répandues, les plus éloignées de tout projet éducatif. Ces pratiques, si elles ont remplacé le Grand Tour, n'en sont pas la suite "naturelle", et leur dimension éducative ne repose pas nécessairement sur un projet explicite.

Une autre voie pour saisir les relations entre tourisme et éducation est ce que l'on appelle le tourisme culturel (visite de musée, du patrimoine, etc.), auquel on accorde une importance grandissante. Ainsi John Urry (2002, p. 137) souligne que le tourisme est aujourd'hui lié à l'apprentissage et reviendrait sur les pas du Grand Tour. Il justifie cela à partir de la popularité des musées, des reconstitutions historiques hyperréalistes. À cela s'ajoutent le désir d'apprendre de nouveaux sports à l'occasion des vacances, le développement du tourisme centré sur la culture et l'art, l'intérêt pour des sites industriels. Le tourisme est donc une "pratique culturelle" (Rojek et Unry, 1997, p. 5). Ce tourisme culturel, Jennifer Craik (1997, p. 12 I) le définit comme une excursion dans d'autres cultures, d'autres lieux, de façon à apprendre à propos des populations, de leurs modes de vie, de leurs arts. On trouve associées une composante éducative et expérientielle et une idée romantique de la culture. Mais au-delà de ces constats qui justifient 
de poser des questions relatives aux apprentissages, on ne trouvera pas de recherche pour étudier de tels apprentissages. La recherche s'est davantage tournée d'une part vers l'analyse de l'offre, de la mise en tourisme, d'autre part vers celle de l'expérience touristique que sur ses effets en termes d'apprentissage.

On trouve également d'importantes réflexions sur la nécessité d'apprendre à être touriste, de s'approprier les pratiques du voyage d'agrément (Équipe Mit, 2002, 2011 ; Ceriani, Knafou, Stock, 2004 ; Ceriani, Duhamel, Knafou et Stock, 2005). Cette dimension est au centre des travaux d'Orvar Löfgren (1994), qui souligne à la fois les transformations collectives et l'apprentissage relatif de compétences sans lesquelles le tourisme ne serait pas possible. Si ces différents travaux font valoir la présence d'apprentissages nécessaires au "devenir touriste", la façon dont on apprend reste une boîte noire, preuve en est la référence, à deux reprises, à l'école dans le dernier ouvrage de l’Équipe Mit (20। I, p. 1 I 8 - |19), même si cela relève de la métaphore. La difficulté est bien là, comprendre comment on apprend quand il n'y pas d'école.

L'éducation populaire à travers diverses formes de tourisme militant, syndical, associatif ou social a développé un projet éducatif au sein du tourisme populaire. Sylvain Pattieu (2009), dans son étude historique de l'association Tourisme et Travail, a montré l'importance et la persistance d'un projet qui conduit à proposer aux classes populaires de pro- fiter de leurs vacances certes pour se reposer, mais également, du fait de l'ambiguiité même du projet, pour accéder à la culture légitime ou prendre conscience de leur situation d'exploitation. Mais le projet est loin de recouvrir la réalité qui a souvent conduit à un alignement sur les pratiques touristiques de masse proposées par le secteur privé. Nous verrons que, malgré la difficulté de maintenir de tels projets d'éducation populaire, il en reste aujourd'hui des éléments.

Enfin, la question de la rencontre interculturelle conduit plus souvent à évoquer le choc culturel que l'apprentissage. Des analyses plus fines montrent sa présence dans certains lieux, mais sans mettre l'accent sur l'apprentissage, même si l'on peut considérer qu'il est sous-jacent (Jack et Phipps, 2005). Tout se passe comme si l'image même du tourisme conduisait à rendre difficile de l'associer à l'apprentissage. Explorer les pratiques touristiques comme espace d'apprentissage représente donc un défi théorique (nos théories sontelles adaptées ?), et suppose d'explorer un domaine bien peu fréquenté.

\section{UNE RECHERCHE}

\section{SUR LE TOURISME SOCIAL}

Nous ne pouvons aller plus loin sans évoquer l'un des terrains ${ }^{(3)}$ sur lequel nous avons travaillé pour mettre à l'épreuve et surtout adapter la théorie. Ainsi que nous l'avons évoqué, rendre visible l'apprentissage de la posture du touriste étant dif- ficile, nous nous sommes tourné vers des populations exclues du tourisme, confrontées à une expérience nouvelle et, pour elles, rare.

Nous avons suivi un groupe de femmes et leurs enfants pris en charge par un centre social d'une ville du Nord de la France (séjour subventionné à $90 \%$ par les caisses d'allocations familiales). Ces personnes sont accompagnées au niveau $\mathrm{du}$ projet et du choix collectif du lieu de vacances et de son programme par leur centre social et, spécifiquement, deux travailleurs sociaux ; le déplacement sur le lieu du séjour (un village de vacances du tourisme social) s'effectue collectivement à l'aide d'un car également utilisé pour les déplacements et visites. Il s'agit bien pour elles de vacances, plus de l'ordre de la villégiature que du tourisme (Urbain, 1994), mais cette différence n'est pas considérée ici comme pertinente, car la mobilité qui en fait une activité touristique prend deux formes : l'éloignement du domicile pour sept nuits, qui suppose un voyage de plusieurs heures, et les excursions réalisées lors du séjour, qui en font un temps de mobilité continuée même si toutes les nuits se déroulent en un même lieu. Pour reprendre l'analyse proposée par Urry (2002), il s'agit bien d'une rupture avec la vie quotidienne et ses lieux.

Notre question est celle des apprentissages de ces personnes aidées pour leurs activités touristiques. Qu'apprennent-elles? Comment apprennent-elles ? Ontelles le sentiment d'apprendre? Y 
a-t-il une spécificité de cette population en matière d'apprentissages touristiques? Quelle théorie devonsnous adapter ou construire pour rendre compte de ces apprentissages ?

Pour répondre, ou tout au moins commencer à construire une réponse, nous nous appuyons sur des données recueillies en 2009 : d'une part, une observation participante immersive dans un village de vacances de la côte atlantique géré par un organisme de tourisme social ${ }^{(4)}$, d'autre part, un entretien collectif réalisé quelques mois plus tard au centre social.

L'observation porte sur neuf familles, sans présence d'aucun père, que la famille soit monoparentale ou, dans deux cas, que le père n'ait pas pu (ou voulu ?) se libérer. Deux des mères sont immigrantes ou filles d'immigrantes (Maroc et Algérie) et témoignent d'une mobilité plus forte $\mathrm{du}$ fait d'un retour périodique dans leur pays d'origine (une année sur deux), où l'une d'entre elles consacre parfois une partie du temps à un séjour en hôtel, le reste se faisant sous forme d'hébergement familial. Les sept autres mères donnent plutôt le sentiment d'une absence de mobilité (pas de voiture ni de permis de conduire, pas de moyens pour des vacances, impression d'enfermement dans leur quartier). Ces familles comptent deux à quatre enfants chacune, avec, dans un cas, la présence de deux jeunes frère et sœur de la mère. Leur discours montre que même un séjour en camping sur la côte de la mer du Nord, à une centaine de kilomètres de leur résidence, apparaît comme inaccessible pour des raisons économiques évoquées, mais aussi devant la difficulté d'organiser un déplacement familial.

Nous avons suivi les activités du groupe, aussi bien celles se déroulant au centre ou à sa proximité (repas, piscine, moments informels, plage) que les excursions en car (souffleur de verre, moulin à farine reconstitué, zoo façon réserve). À cette occasion, nous avons eu de nombreuses discussions informelles. Trois mois plus tard, nous avons organisé un entretien collectif avec quatre femmes fréquentant le centre social accompagnateur du séjour. Deux d'entre elles avaient participé au séjour évoqué, deux autres à des séjours semblables mais en d'autres lieux ${ }^{(5)}$.

\section{PROJETS ET SPÉCIFICITÉ ÉDUCATIVE DU TOURISME SOCIAL}

S'intéresser à ce type de tourisme signifie rencontrer le projet éducatif, ou plutôt les projets éducatifs, du centre social d'une part, de l'association gestionnaire du centre de vacances d'autre part. Celle-ci définit son projet de vacances familiales comme permettant "de se retrouver, de partager du temps et de se ressourcer tout en s'ouvrant aux antres"; les activités "s'appuient sur un concept à la fois ludique et éducatif". Les séjours se doivent d'être "vecteurs de mixité sociale et d'éducation à la citoyenneté". Il s'agit de "développer les relations entre vacanciers, générer la convivialité, développer du lien social” et de découvrir l'environnement naturel, humain et culturel.
Ce tourisme social s'inscrit dans une perspective d'éducation populaire. Comme le souligne Luc Greffier, les projets éducatifs restent présents, mais se heurtent souvent à une pratique considérée comme "consumériste" des vacanciers. Ainsi, "les deux opérateurs issus du mouvement ouvrier [étudiés par l'auteur] sont interpellés par la tension repérée entre la demande touristique de populations de plus en plus marquées par une culture partagée des vacances et le projet social de nature collective mais non collectiviste qu'ils défendent” (Greffier, 2006, p. 182), et “on peut donc traduire au sein du panel retenu [quatre centres de vacances de la région Aquitaine] une forte tension entre les idéologies soutenues par les mouvements historiques $d u$ tourisme social et les réalités partagées d'une culture des vacances de plus en plus construite sur un mode de consommation. Cette tension discrimine les opérateurs qui défendent un engagement historique politique du tourisme social et ceux qui s'inscrivent comme de simples acteurs dans un traitement des politiques sociales du tourisme” (ibid., p. 183). L'affirmation éducative semble plus forte, plus facile à mettre en œuvre et plus évidente quand il s'agit d'enfants. Pour les adultes, cette démarche éducative est très largement liée au développement d'animations ludiques, sportives et festives. À cela s'ajoutent des programmes de découverte régionale, mais l'investissement des vacanciers dans de telles pratiques est très faible (p. 193). 
On retrouve ici les constats de Sylvain Pattieu sur la rupture entre le projet éducatif de Tourisme et Travail et les attentes de touristes prêts à "bronzer idiots", à tel point que la réussite supposée de celui-là à travers une prise de conscience politique apparait comme un leurre (Pattieu, 2007, p. I0I).

C'est dans le cadre d'une tension déjà ancienne entre projet éducatif et logique touristique que se déroule le séjour observé. Mais notre population possède sans doute une caractéristique propre qui justifie l'aide de la CAF, pour laquelle "les vacances et les loisirs jouent un rôle essentiel dans l'insertion sociale" (Greffier, 2006, p. 59). Du point de vue du centre social comme des villages, cette population est considérée comme ne maîtrisant pas les codes du tourisme dans le cadre des séjours collectifs. Mais cela ne relève que pour partie de la question du tourisme : les dimensions de la vie collective et de la parentalité sont le plus souvent évoquées. Il s'agirait de familles marquées par des lacunes dans les compétences parentale et éducative, par une difficulté à accepter les règles de la vie collective (en ce qui concerne le repas, le repos nocturne, etc.).

Cela renvoie à un premier constat, qui permet de souligner la difficulté de la question des apprentissages touristiques : les pratiques touristiques ne sont que partiellement des pratiques spécifiques. Le touriste transporte avec lui des façons d'être, des modes de vie, des routines quotidiennes. Henry Raymond (1982) évoque un "transfert symbolique de la vie quotidienne” (p. 10). Mais ces pratiques quotidiennes sont sorties de leur contexte habituel et, dans certains cas, quand les lieux de séjour sont fréquentés par une population hétérogène, rendues visibles à d'autres qui peuvent ne pas les partager. Le tourisme met en contact des populations qui n'ont pas l'habitude de vivre ensemble. Le plus souvent, cette question est évoquée à travers le contact entre le touriste et l'autochtone; dans notre cas, il s'agit du contact entre touristes de même origine en vacances dans leur propre pays, ainsi que du décalage entre ces mêmes populations et les professionnels qui les encadrent (Ulmann, 20I I). Les pratiques touristiques n'apparaissent pas comme étant "pures" et séparées, elles greffent des pratiques de mobilité sur des pratiques quotidiennes incorporées que chacun transporte avec soi. C'est sans doute leur spécificité, par rapport à d'autres pratiques de loisir (comme le jeu) qui peuvent être pensées sur la base de la séparation, de la distinction avec les pratiques quotidiennes. Les pratiques touristiques sont fortement liées au fait de transporter, de déplacer (et parfois de mettre en péril) les pratiques quotidiennes, avec des stratégies différentes selon les touristes (ou leur apprentissage du tourisme), qui peuvent tendre à la volonté de reproduire au plus près la vie quotidienne ordinaire ou, au contraire, de transformer celle-ci pour adopter des pratiques spécifiques.

On trouve cette tension dans notre groupe entre la volonté de maintenir le régime alimentaire habituel (en particulier pour les enfants) et celui d'avoir des expériences liées aux produits locaux ou à la festivité du restaurant comme symbole de la rupture vacancière. La demande de ne pas nettoyer la chambre à la fin du séjour n'est pas respectée par ces femmes dont plusieurs nous ont dit combien il était important pour elles de faire le ménage et de rendre leur chambre propre, mais aussi de retrouver les routines du quotidien : "Comme on a l'habitude par exemple de faire à manger, de faire le ménage, de faire les lits, de faire plein de trucs - et qu'on voit que... et qu'on peut pas les faire... ça commence à embêter un peu."

Les pratiques touristiques apparaissent comme une altération, une modification plus ou moins importante de pratiques du quotidien, une "dé-routinisation" (Ceriani et al., 2008) recherchée ou subie. Leur nonmaîtrise conduit à reprendre ou à maintenir les façons de faire au plus près des pratiques habituelles. Le tourisme se joue dans cette différence entre volonté de maintenir et reproduire dans un autre contexte les pratiques du quotidien et celle d'adopter des pratiques spécifiques et différentes. Le conflit (lors d'une autre observation) peut passer entre enfants et parents, quand ces derniers cherchent à maintenir une logique du quotidien (prendre les repas à midi) alors que, pour les jeunes, les vacances se caractérisent par l'abandon de toute référence horaire, se coucher tard, se lever tard, ne plus 
avoir d'heure pour manger. Il est intéressant de constater que ce sont les parents ayant une plus grande expérience touristique qui acceptent pour leurs enfants adolescents une redéfinition complète du rythme (avec, en contrepartie, une autonomisation de ceux-ci).

Autre dimension du séjour, les pratiques cachées dans la famille deviennent visibles (en particulier les pratiques éducatives) ; elles constituent un champ d'observation et de découverte. On entend des discours critiques des autres pratiques, mais aussi certains entretiens évoquent l'idée que l'on peut apprendre des pratiques éducatives des autres. L'attachement aux enfants, souvent évoqué, est fondamental dans la population rencontrée et se traduit par le désir d'une relation maintenue et permanente (des enfants toujours sous leur regard), le refus d'utiliser les structures de garde du centre (sauf exception nécessaire). Non seulement les enfants sont au cœur des vacances, mais le discours conduit à mettre en avant un ethos de bonne mère, renforcé par la visibilité (et la critique) des pratiques familiales des autres vacanciers. Les vacances conduisent à être parent sous le regard des autres, à se mettre en scène comme parent, et peuvent développer une réflexivité sur ce que signifie être parent, mère en l'occurrence pour notre population. On peut voir fonctionner ainsi une instance de normalisation par la collectivité.

La conjonction de ces deux éléments conduit à un projet de nor- malisation sociale par le tourisme. Il s'agit moins d'apprendre à ces familles à être touristes que de profiter de la situation touristique, qui rend visibles ces pratiques familiales habituellement cachées, pour intervenir sur leur mode de vie et sur l'éducation parentale. Le projet des vacances devient un projet d'intégration de la norme sociale et familiale dominante.

Minnaert, Maitland et Miller (2009) montrent, à partir de leurs données recueillies en Angleterre, l'efficacité sociale du tourisme dans la transformation des modes de vie, c'est-à-dire l'incorporation du modèle dominant. Ils mettent en évidence la dimension normative que les vacances peuvent avoir au sein de la famille.

Dans le cas de notre centre social, on demande aux participants, dans la construction du programme des activités prévues en amont, de penser aux enfants. Ainsi la visite du zoo a-t-elle été choisie en fonction de ceux-ci. Il s'agit bien de viser l'éducation familiale à travers l'activité de tourisme, même si le projet ne se limite pas à cela et inclut la dimension de souffler, de mettre à distance la vie quotidienne, de se ressourcer.

Le paradoxe du village de vacances à vocation (voulue ou de fait) sociale est d'être un lieu de visibilité des pratiques quotidiennes et familiales (une forme de panopticon ${ }^{(6)}$ où chacun agit sous le regard de l'autre). Quand les familles ne sont pas considérées comme prêtes pour être des touristes, il s'agit moins de viser l'apprentissage de la pratique touristique, pas vraiment perçue, facilement naturalisée, que de stigmatiser des modes de vie considérés comme peu compatibles avec la vie collective : cela signifie respecter les autres, afficher une éducation familiale conforme aux normes.

Au-delà de la critique que l'on peut porter sur cette logique de normalisation, celle-ci ne révèle-t-elle pas l'articulation entre les compétences du tourisme et des compétences sociales plus générales qui peuvent conduire à deux remarques? 1. Le tourisme est de fait réservé à ceux qui sont capables de développer des comportements considérés comme adaptés à la vie collective. Il y aurait des pré-requis au tourisme, qui en réserveraient la pratique à ceux qui maîtrisent déjà d'autres types de pratiques qui n'ont rien de spécifique. 2. Apprendre à être touriste, c'est apprendre des pratiques hybrides dont une partie ne relève pas de la spécificité touristique mais d'autres dimensions de la vie sociale et collective. Le tourisme est ici une pratique qui révèle d'autres apprentissages sociaux fondamentaux, et un espace qui permet des apprentissages plus larges que la pratique touristique, tout particulièrement quand il s'agit de populations considérées comme défavorisées.

\section{LES APPRENTISSAGES TOURISTIQUES}

Cependant, l'apprentissage du tourisme, quoique moins nettement visible, est bien présent dans nos 
observations, plus que dans les entretiens. Et, d'une certaine façon, c'est bien de cela qu'il s'agit, ne seraitce que parce que la règle du centre social est de limiter l'accompagnement à deux séjours en groupe, après quoi il s'agit tout au plus de soutenir les départs individuels. Pour les travailleurs sociaux qui accompagnent les neuf familles observées, il manque à ces dernières "la capacité d'organisation" nécessaire à un séjour de vacances.

Comme le souligne Barry Brown (2007), le touriste, tout au moins dans une situation individuelle, doit résoudre un certain nombre de problèmes pour organiser son séjour, tout au moins dans un lieu nouveau pour lui. Il doit décider quoi faire, comment le faire, où le faire et comment atteindre le lieu. Cette logique d'organisation et de résolution de problèmes est d'autant plus centrale que le loisir touristique est un temps vide qui peut être empli par une multitude d'activités différenciées. Il ne peut être organisé de l'extérieur comme le sont nombre de tâches liées au travail. C'est justement cette absence de capacité à résoudre de tels problèmes et à organiser ses vacances qu'évoquent les responsables du centre social.

Pour résoudre ces problèmes les touristes individuels mettent en œuvre différentes stratégies, telles que le partage avec d'autres touristes, l'usage des guides (livres) ou d'autres dispositifs trouvés sur place, le recours aux cartes, la planification préalable à la visite (Brown, 2007). C'est cet ensemble de tâches que le centre social va prendre en charge ou tout au moins accompagner, en planifiant séjour et visite avant le départ avec les vacanciers, en s'occupant de l'orientation sur place (trouver les lieux et les informations sur les horaires, lire les cartes), en soutenant les différents projets nés sur place (achats, feu d'artifice).

L'activité du centre social consiste à apprivoiser l'inconnu propre à un tel séjour et à limiter l'incertitude. Il met en place un dispositif qui devrait à terme permettre aux populations d'atteindre une autonomie en matière de pratiques touristiques. Il s'agit bien de l'apprentissage de telles pratiques.

Le séjour est alors un apprentissage des vacances, d'autant plus effectif que les populations ont une expérience qui dépasse peu l'excursion ponctuelle ou le court séjour en camping dans des localités proches, à l'exception des deux mères immigrantes dotées de compétences "mobilitaires"mais ayant quelques difficultés, ne serait-ce que d'ordre économique, à les exercer en France. À partir de nos données, nous pouvons, dans le cadre d'un séjour d'une semaine dans un village de vacances, repérer différents apprentissages :

- la conquête de l'espace environnant et de la plage accessible à pied (2 à 5 minutes). Repos, jeux avec les enfants (très surveillés), détente et plaisanteries entre adultes ; apprendre aussi à se protéger du soleil ;

- la vie dans le centre de séjour, notamment la nourriture (mais cette dimension est happée par le quoti- dien, la lessive étant un important sujet de discussion et de problèmes); - les visites, fort appréciées (même par celles qui se montraient réservées) ; un souffleur de verre, un moulin reconstitué selon les techniques anciennes de construction, un zoo façon safari, visités collectivement en car. Elles constituent un élément important de la performance touristique : on écoute (on demande aux enfants d'écouter), on regarde, on achète à la fin ;

- le restaurant collectif payé par le centre social sur le budget chèquesvacances, moment symbolique fort (coquetterie, rêve de prince charmant pour les mères célibataires, etc.) ; - quelques activités comme le spa, la visite du marché, les apéros-jeux, le feu d'artifice. Dans ce dernier cas, il s'agissait de s'y rendre à pied, le long de la mer, et les femmes nous ont dit qu'elles n'y seraient pas allées sans le soutien des travailleurs sociaux, craignant la foule pour leurs enfants.

\section{UNE PARTICIPATION GUIDÉE}

Il y a donc une réelle participation aux pratiques touristiques à travers, d'une part, l'adaptation (parfois difficile) des pratiques quotidiennes à la situation du séjour de vacances et, d'autre part, le développement de pratiques spécifiquement touristiques, telles qu'elles sont construites en partie à travers l'accompagnement par le centre social. C'est de l'ordre de la participation, au sens d'engagement dans une activité partagée (Wenger, 2005). 
Mais ce qui caractérise cette population est que sa participation est guidée. La notion de "participation guidée” a été développée par Barbara Rogoff (1990) pour mettre en évidence les modalités d'apprentissage et de développement de l'enfant, à travers la participation et le soutien des autres (adultes, pairs) ; mais il n'y a pas de raison de limiter cette notion aux enfants, dans la mesure où, pour Rogoff elle-même, il n'y a pas de différence de modalités d'apprentissage entre enfants et adultes, à cela près que ces derniers, du fait de leur expérience antérieure incorporée, peuvent avoir plus de difficultés à apprendre - comme on peut le noter pour l'apprentissage d'une langue étrangère (Brougère, 2009, pp. 276-277). Le guidage peut être explicite ou tacite, et renvoie à une activité conjointe et partagée avec un expert ou au moins une personne plus compétente dans le domaine visé par la pratique. Il s'appuie sur des communications plus ou moins tacites, mais aussi sur l'engagement de celui qui apprend dans l'activité, directement, par imitation ou à travers l'observation. C'est en participant que l'on apprend, et c'est la participation qui est guidée, sans que l'apprentissage soit une activité séparée, isolée ; il est totalement intégré à la participation et donc souvent invisible. C'est affaire d'interdépendance et d'autonomie socialement construite. C'est le moyen de s'approprier les pratiques, mais dans un processus de transformation de celles-ci : “[...] L'appropriation des pratiques sociales relève d'un processus créatif. En ce sens les informations et habiletés ne sont pas transmises mais transformées dans le processus d'appropriation" (Rogoff, 1990, p. 197) $)^{(7)}$. La participation guidée renvoie non seulement au processus de communication mais aussi aux arrangements des pratiques, pour les rendre accessibles et appropriables.

Dans nos observations concernant le tourisme social, nous découvrons différentes modalités de guidage : faire un programme, accompagner, organiser, orienter, résoudre les problèmes et les conflits (avec les autres vacanciers, avec le personnel du centre). Ainsi la préparation, qui s'est déroulée en plusieurs séances au centre social, a conduit à la programmation de visites malgré le peu d'enthousiasme a priori, mais, dans une perspective d'éducation populaire, les responsables y tiennent. L'intérêt ici est que les réticences sont largement levées au moment des visites, appréciées et intégrées comme un moment fort de la pratique touristique ainsi appropriée. Les travailleurs sociaux sont là en permanence dans le village de vacances, disponibles pour régler les conflits avec la direction quand des enfants sont trop bruyants le soir, pour accompagner le groupe la première fois à la plage, l'aider à organiser une demi-journée d'achats, surveiller les enfants dans l'atelier du souffleur de verre.

Le discours sur les deux travailleurs sociaux témoigne de l'importance de ces personnes, et de la relation affective forte qui se noue avec ces “guides”. C'est un apprentissage sans enseignants ni pédagogie, les travailleurs sociaux guidant la pratique à laquelle ils participent eux-mêmes. En cela, il n'est pas visible car celui qui apprend (ici les pratiques touristiques) ne fait que participer à une activité pour laquelle il est aidé, soutenu, guidé. Il n’y a pas décalage entre la pratique et son apprentissage, c'est en pratiquant que l'on apprend, mais la situation révèle nettement le processus de guidage.

L'hypothèse que nous formulons ici est que la situation de tourisme social aidé permet de voir ce qui est vrai de façon plus large, à savoir que l'apprentissage des pratiques touristiques relève pour une grande part de la participation guidée : - selon le modèle de Rogoff, guidage des parents et d'autres adultes vis-à-vis des enfants ; c'est ce qu'évoque l'Équipe Mit (20II) à travers l'idée de transmission. Il nous semble que la notion de participation est plus juste ; - pour les adultes, on peut saisir le rôle des guides, au sens usuel dans le tourisme, et d'autres accompagnateurs ;

- les guides écrits sont des éléments essentiels de cette participation guidée ;

- on peut découvrir d'autres dispositifs moins évidents de guidage : office du tourisme, hôtelier, propriétaire du gîte ou de la chambre d'hôte, autres vacanciers (les habitués). Jack et Phipps (2005) montrent bien comment les touristes 
échangent des informations, des histoires entre eux et ainsi orientent réciproquement leurs activités.

À travers la participation guidée, le touriste (ou celui qui devient touriste) s'approprie les pratiques touristiques, mais au sens où il les transforme, les interprète sans nécessairement les reproduire fidèlement. Ce processus de socialisation touristique est bien un processus d'apprentissage.

\section{L'EXPLORATION GUIDÉE}

Si en participant aux pratiques touristiques, on apprend, ce n'est pas le seul type d'apprentissage que l'on peut mettre en évidence, même s'il s'agit du plus facile à repérer, d'autant plus que l'on suppose qu'il doit se faire à un moment ou un autre de la vie du touriste. D'autres dimensions de l'apprentissage peuvent être perçues, en particulier par le touriste lui-même. Ainsi, un sentiment d'apprendre quelque chose émerge dans les entretiens : "Ben déjà on voit des choses qu'on n'a pas l'habitude de voir, et puis il y a les autres personnes, c'est vrai que le contact avec les autres personnes qu'on connaît pas, c'est vrai que parler avec les autres personnes [...]."

À travers la découverte de lieux et de personnes, la rencontre avec de l'inconnu et des inconnus apparaît une dimension de l'apprentissage qui semble difficilement relever de la participation. Certes la personne continue à participer à des pratiques touristiques, mais nous sommes loin de l'idée de communauté de pra- tique, sauf à évoquer une communauté virtuelle des touristes, ce qui ne correspond pas à la dimension concrète d'un faire ensemble présente dans la notion développée par Wenger (2005).

Il semble qu'ici il soit intéressant de renvoyer à une logique d'exploration, d'exploration curieuse (Jacob, 2002) liée à la disponibilité, en particulier dans le cadre du divertissement ou en réponse à l'ennui, liée à un état d'excitation (Auray, 20 I I). Le thème de l'émerveillement rencontré dans notre entretien collectif nous semble renvoyer à cela. Ainsi, au sujet de la visite d'une chocolaterie à l'ancienne ("on n'imagine pas que vraiment dans les anciens temps tout se faisait par main") : "J'étais émerveillé parce que c'était vraiment, on voyait que c'était vraiment... c'était fait d'eux-mêmes." On peut le savoir par d'autres canaux, mais :"Quand on le voit, ça, par contre, ça m'a vraiment touchée..." Cet émerveillement, d'autant plus fort qu'il renvoie à une rupture avec le quotidien, avec l'ordinaire de la vie, vient caractériser cette expérience. Enfants et adultes ont été émerveillés. Cela conduit à penser que la force de l'expérience touristique ne se mesure pas de façon absolue en fonction de la nature de ce qui est vécu, mais de façon différentielle, en fonction du degré de rupture ressenti avec le quotidien. La discontinuité est ici forte dans notre population et rend encore plus visibles les apprentissages potentiels.

L'émerveillement renvoie à l'idée de voir "pour de vrai". Lors de la visite d'un souffleur de verre, "c'est mieux qu'à la télé", dit une des mères, "... maintenant on sait comment c'est fait" ; cette dimension d'apprentissage immédiat est évidente et appréciée. Ainsi on sait quelque chose, on a vu en vrai (même si on avait déjà vu à la télé), le vrai n'étant pas l'authentique qui pourrait renvoyer à une théorie du tourisme comme recherche de l'authenticité dans un monde aliéné, discutée par Cohen (1979). En effet, voir un parc Disney est du même ordre et, pour notre terrain d'observation, le zoo safari avec son village africain est considéré comme vrai. Il n'y a pas de référence à une vérité ontologique, mais à quelque chose qui est là sans médiation, même s'il s'agit d'un artefact construit pour le touriste (comme le moulin à farine visité). Il s'agit bien "d'une expérience originale sans rapport avec la découverte $d u$ Monde par le truchement des médias : d'une confrontation à la réalité, non dénuée de représentations bien sûr, mais moins médiatisée" (Ceriani et al., 2008).

Le vrai est à définir dans une logique de loisir comme découverte. Cela renvoie à une dimension essentielle du tourisme, dont l'Équipe Mit (20I I) souligne l'importance à travers les trois mots "voir, découvrir et s'émerveiller" (p. | | I). Le tourisme permettrait de voir pour de vrai (et non à travers la représentation médiatisée). On peut ainsi voir des reconstitutions historiques (fabrication du chocolat), des activités artisanales à l'ancienne (souffleur 
de verre), des animaux "en liberté" (zoo façon safari), etc. Voir "pour de vrai" semble être une dimension importante, qui définit bien le tourisme par opposition au spectacle télévisuel, celui de l'immersion dans le monde, qui permet un contact corporel et visuel : "[...] La pratique touristique est une pratique in situ, avec présence $d u$ corps $d u$ touriste auprès d'autres corps" (Équipe Mit, 20II, p. 275).

Le regard mis en avant par Urry (2002) n'en constitue qu'un aspect, peut-être pas le plus important pour des vacanciers populaires dont d'autres études soulignent l'attrait pour des festivités collectives (Périer, 2000). Cela donne une force à la connaissance par contact. Le tourisme serait le loisir qui s'oppose à la télévision et la complète. La télévision ne remplace pas le voyage, mais au contraire donnerait envie de voir. Cela apporte à l'apprentissage une dimension corporelle : apprendre par corps, utiliser sa présence comme moyen de connaître, qu'il s'agisse de compléter par cette confrontation des connaissances antérieures ou de plonger dans un nouvel univers à explorer, pour autant que la curiosité soit là.

L'entretien collectif montre que la question de l'apprentissage n'est pas difficile à solliciter même si elle prend la figure que nous venons d'évoquer. Elle s'intègre, mais avec son exceptionnalité, dans les apprentissages du quotidien, qui consistent à raconter aux enfants de quoi est fait le monde, elle entre en synergie avec des apprentissages scolaires actuels pour les enfants ou oubliés pour les mères. Autre source d'apprentissage évoquée, les discussions avec les autres vacanciers, des personnes "que l'on ne connaît pas d'avance", qui habitent des régions que l'on n'a pas visitées. À l'émerveillement que l'exceptionnel produit se combine la curiosité du vacancier, qui se traduit par la manifestation d'un intérêt pour ce qui est différent, à condition sans doute que cela ne soit pas trop différent.

L'exploration renvoie bien à l'altérité et à l'inconnu propres au tourisme (Équipe Mit, 20।l). Et, indéniablement, nos familles explorent la plage, les lieux transformés en attraction touristique, le village de vacances en tant qu'il associe espace et personnes inconnues.

De même que nous avons pu parler de participation guidée pour l'apprentissage des pratiques touristiques elles-mêmes, nous pouvons ici parler d'exploration guidée : un ensemble de dispositifs organise le tourisme pour permettre, par le guidage, la découverte. Cela va du dispositif créé ou adapté pour le touriste à tous les supports sur lesquels peut compter le touriste sans qu'ils lui soient exclusivement destinés. On peut distinguer des dispositifs d'orientation (guides, centres d'information, signalétique) et des dispositifs de découverte (spectacle de flamenco conçu pour les touristes, musées, zoo, centres d'interprétation...).

Dans notre cas, les travailleurs sociaux guident tout autant la participation aux pratiques touristiques que l'exploration de nouveaux espaces sociaux, les deux étant indissociables, la participation supposant des pratiques touristiques effectives qui sont de l'ordre de l'exploration. L'accompagnement au feu d'artifice montre bien comment l'exploration d'une telle manifestation est encadrée, à la demande même des mères inquiètes de ce que cela peut avoir d'incertain, d'incontrôlable, en particulier pour leurs enfants. Mais l'exploration guidée recourt aussi à tous les dispositifs de guidage touristique traditionnels, les brochures, les attractions construites (comme le souffleur de verre, dont l'organisation de l'atelier et le discours guident l'exploration de la fabrication du verre). Il reste que l'exploration conserve une part d'incertitude, de rencontres non programmées, comme celle de cette apprentie souffleuse de verre québécoise, en stage, travaillant chez un souffleur français installé au Québec, ancien voisin du chauffeur de car. De cette coïncidence s'ensuit une conversation sur le Québec, l'immigration des Français, l'artisanat - exploration partagée et nettement moins guidée.

Le touriste est la figure antinomique de l'explorateur, celui qui découvre sans guide mais qui, par son relevé, permettra à d'autres d'être guidés pour découvrir à leur tour ce qu'il a découvert. Le tourisme est par excellence affaire de guide. Mais c'est sans doute ce guidage qui rend possible l'apprentissage en évitant l'anxiété d'une confrontation à l'inconnu qui ne serait pas mâ̂- 
trisable. Pour nos familles le guidage multiple par les travailleurs sociaux, eux-mêmes guidés à l'aide des ressources usuelles du tourisme (guides papier, sites internet, informations transmises par le village de vacances, etc.), permet un contact maîtrisé de l'inconnu, la réduction de l'incertitude et la possibilité d'apprendre. Mais toute guidée qu'elle soit, l'exploration, comme la participation, suppose un engagement de la personne sans lequel il ne peut y avoir exploration ni participation et donc apprentissage. On peut parler d'engagement possible car soutenu par les travailleurs sociaux dans notre cas, les professionnels du tourisme ou d'autres vacanciers. Il faut également, pour reprendre les termes de Billett (2004), que la situation offre une possibilité d'exploration, des affordances. Mais, là encore, les guides (et ici encore, les travailleurs sociaux) aident à percevoir cellesci en les sélectionnant, en les commentant, en cadrant l'activité.

Le tourisme social - avec l'accent mis sur l'aide, l'accompagnement permet de voir de façon très explicite et évidente une logique de guidage à l'œuvre. Il permet également de rencontrer des personnes dont l'expérience touristique est limitée et qui dévoilent plus que d'autres les logiques d'apprentissage sousjacentes, sans pour autant développer une logique de tourisme savant et distingué.

Cela nous a permis de transformer et d'adapter au tourisme des théories préexistantes de l'apprentissage. Si la participation est ici centrale, comme dans toutes les pratiques sociales étudiées sous l'angle de l'apprentissage, elle rejoint explicitement la forme étudiée par Rogoff à propos des enfants en milieu familial, la participation guidée. Cependant cette notion ne permet pas de saisir l'ensemble des apprentissages repérés, ce qui nous a fait retenir la notion d'exploration, certes sortie de l'actualité de la recherche sur l'éducation, mais qui est aujourd'hui convoquée dans les analyses de l'usage d'internet (Auray, 20I I). Il nous semble à nouveau que la spécificité est ici aussi le guidage, ce qui nous conduit à proposer la notion d'exploration guidée comme modalité d'apprentissage mobilisée par les pratiques touristiques.

Participation et exploration guidées ne sont pas spécifiques au tourisme, mais cette activité, à travers l'importance qu'elle accorde à la sortie du quotidien, à la mobilité, à la rupture, met en évidence l'importance du guidage dans la pratique, permettant ainsi l'apprentissage, dans la maîtrise des pratiques, dans la possibilité de tirer d'une situation de nouvelles expériences et connaissances. Selon ce modèle, l'apprentissage n'est pas quelque chose d'extérieur, un effet du tourisme, mais ce qui accompagne la pratique, à la fois pour la rendre possible et comme une dimension de l'expérience qu'elle offre.

NOTES

(I) Les notions d'apprentissage et d'éducation sont loin d'être définies de façon claire, mais nous opérerons la distinction suivante :

"éducation" renverra à un projet, à une mise en forme, alors qu'“apprentissage" renvoie soit aux effets de l'éducation soit à l'absence de mise en forme éducative.

(2) Le terme, forgé par Gibson (1979) dans le cadre de la psychologie de la perception, à partir du verbe anglais to afford (offrir, mettre à disposition), a pour but de conceptualiser les propriétés de l'environnement telles qu'elles sont perçues (l'affordance) en tant que possibilité d'action en relation avec les caractéristiques d'un individu (animal ou humain), à la façon, par exemple, dont la forme d'une branche d'arbre offre une possibilité de s'asseoir à un humain (du fait de la taille de ce dernier, de sa configuration physiologique rapportée à l'arbre). Chez Billett (2004), l'usage est moins précis mais renvoie à cette relation entre ce qu'offre la situation et ce qu'en perçoit (dans un sens plus général et non limité à la perception visuelle) l'individu. Le terme, utilisé dans différentes disciplines telles que l'ergonomie ou le design, n'est pas traduit en français faute de vocable approprié. Nous nous rangeons à cet usage qui consiste à importer directement le terme anglais.

(3) Un autre terrain, que nous n'évoquerons pas ici, renvoie à l'observation de soi comme "tourisme apprenant" (Brougère, 2012).

(4) La recherche présentée a reçu le soutien financier de cet organisme de tourisme social et bénéficié de l'ouverture de ses centres aux chercheurs ainsi que de celle de l'université Paris 13, qui abrite notre équipe de recherche Experice.

(5) On trouvera une présentation plus détaillée de cette recherche dans Brougère (201 I).

(6) Le terme renvoie à l'architecture carcérale imaginée par Jeremy Bentham à la fin du XVIII siècle, permettant à un gardien de voir constamment prisonniers et cellules depuis la tour centrale où il se trouve.

(7) Notre traduction. 


\section{RÉFÉRENCES BIBLIOGRAPHIQUES}

Nicolas Auray, "Les technologies de l'information et le régime exploratoire", dans Danièle BOURCIER et Pek VAN ANDEL (dir.), La Sérendipité : le hasard heureux, Hermann, 201 I, pp. 329-342.

Vincent BERRY, "Loisirs numériques et communautés virtuelles : des espaces d'apprentissage", dans Gilles Brougère et Anne-Lise ULMANN (dir.), Apprendre de la vie quotidienne, Puf, 2009, pp. I43153.

Stefen BILLETT, "Working participatory practices: conceptualising workplaces as learning environments", Joumal of Workplace Learning, vol. 16, n 5-6, 2004, pp. 312-324.

Jean Boutier, Le Grand Tour: une pratique d'éducation des noblesses européennes (XVIe-XVIII siècles), 2004 [en ligne]. À partir de http://halshs.archives-ouvertes.fr/docs/00/05//0/63/PDF/Boutier_J.2004-GrandTour.pdf [consulté le 9 mars 20 I I].

Gilles Brougère, Jeu et éducation, L'Harmattan, 1995.

Gilles Brougère, Jouer/Apprendre, Economica, 2005.

Gilles Brougère, "Une théorie de l'apprentissage adaptée : l'apprentissage comme participation", dans Gilles BROUGÈRE et Anne-Lise ULMANN (dir.), Apprendre de la vie quotidienne, Puf, 2009, pp. 267-278.

Gilles Brougère, "Qu'apprennent des personnes aidées en vacances ?"', dans Actes du congrès de l'Actualité de la recherche en éducation et en formation (AREF), Université de Genève, septembre 2010, 2011 [https://plone2.unige.ch/aref2010/symposiumslongs/coordinateurs-en-b/tourisme-et-

apprentissages/Quapprennent\%20des\%20personnes\%20aidees\%20 en\%20vacances.pdf/view].

Gilles BROUGÈRE, "S'observer comme touriste apprenant", communication au colloque Tourisme et apprentissages (Université Paris 13, avril 2011), Experice, 2012 [http://www.univ-

paris | 3.fr/experice/fr/actes-coll0 //brougere.html].

Gilles Brougère et Hélène BÉZILLE, “De l'usage de la notion d'informel dans le champ de l'éducation", Revue française de pédagogie, $n^{\circ}$ 158, 2007, pp. |17-160.

Gilles Brougère et Anne-Lise UlmaNN (dir.), Apprendre de la vie quotidienne, Puf, 2009.

Barry BROWN, "Working the problems of tourism", Annals of Tourism Research, vol. 34, n 2, 2007, pp. 364-383.

Giorgia Ceriani, Rémy KNAfOU, Mathis Stock, “Les compétences cachées du touriste", Sciences Humaines, n |45, 2004, pp. 28-31. Giorgia Ceriani, Philippe Duhamel, Rémy Knafou, Mathis Stock, "Le tourisme et la rencontre de l'autre", L'Autre : cliniques, cultures et sociétés, vol. 6, n I, 2005, pp. 71-82.

\section{Giorgia CERIANI, Vincent COËFFÉ, Jean-Christophe GAY, Rémy}

KnAfou, Mathis Stock et Philippe Violier, "Conditions géogra-

phiques de l'individu contemporain", EspacesTemps.net, 2008

[http://www.espacestemps.net/document4573.html].

Erik COHEN, "A phenomenology of tourist experiences", Sociology, vol. 13, 1979, pp. |79-20।.

Erik CoHeN, “Tourism as play”, Religion, n I5, 1985, pp. 29|-304. Jennifer CRAIK, "The culture of tourism", dans Chris ROJEK et John URRY (dir.), Touring Cultures. Transformations of travel and theory,

Routledge, 1997, pp. I13-136.

ÉQUiPE MIT, Tourismes I. Lieux communs, Belin, 2002.

ÉQUIPE MIT, Tourismes 3. La révolution durable, Belin, 201 I.

James J. GiBson, The Ecological approach of visual perception,

Lawrence Erlbraum, 1979.

Barney G. Glaser et Anselm A. Strauss, La Découverte de la théorie ancrée, Armand Colin, 2010.

Luc GrefFIer, L'Animation des territoires. Les villages de vacances du tourisme social, L'Harmattan, 2006.

Gavin JACK et Alison PHIPPS, Tourisme and Interculturel Exchange. Why Tourisme Matters, Channel View Publications, 2005.

Stéphane JACOB, La Curiosité. Éthologie et psychologie, Mardaga, 2002.

Derek LAYDER, Sociological practice. Linking theory and social research, Sage, 1998.

Orvar LÖFGREN, "Learning to be a tourist", Ethnologia Scandinavica, vol. 24, 1994, pp. 102-125.

Jean LAVE et Etienne Wenger, Situated Leaming. Legitimate Peripherical Participation, Cambridge University Press, 1991.

Lynn MinNaert, Robert Maitland et Graham Miller, "Tourism and social policy, the value of social tourism", Annals of Tourism Research, vol. 36, n², 2009, pp. 316-334.

Richard D. MITCHELL, "Learning through play and pleasure travel: using play literature to enhance research into touristic learning", Current Issues in Tourism, vol. I, n², 1998, pp. 176-188.

Sylvain Pattieu, "Nous n'avons rien à Katmandou. Production mili- 
tante et usages populaires du tourisme", Actes de la recherche en sciences sociales, $n^{\circ}$ 170, 2007, pp. 88-101.

Sylvain PAtTieu, Tourisme et travail. De l'éducation populaire au secteur marchand (1945-1985), Les Presses de Sciences Po, 2009.

Pierre Périer, Vacances populaires. Images, pratiques et mémoires, Pur, 2000.

Thomas G. POWER, Play and Exploration in Children and Animals, Lauwrence Erlbaum, 2000.

Henri Raymond, "Tourisme social et loisirs. L'espace des alternatives", Temps libre, n 5, 1982, pp. 101-109.

Barbara Rogoff, Apprenticeship in Thinking : Cognitive Development in Social Context, Oxford University Press, 1990.

Chris RoJEK et John URRY, "Transformation of travel and theory", dans Chris RojEK et John URRY (dir), Touring Cultures. Transformations of travel and theory, Routledge, 1997, pp. I- 19.

Daniel SCHUGURENSKY, "Vingt mille lieues sous les mers : les quatre défis de l'apprentissage informel", Revue française de pédagogie, $n^{\circ}$ 160, 2007, pp. 13-27.

Brian SUTTON-SMITH, The Ambiguity of play, Havard University Press, 1997.

John TOWNER, "The Grand Tour : a key phase in the history of tourism", Annals of Tourism Research, vol. 12, n 3, 1985, pp. 293333.

Anne-Lise UlmanN, "Les professionnels du tourisme social : des modes d'agir contre la barrière culturelle", dans Actes du congrès de l'Actualité de la recherche en éducation et en formation (AREF),

Université de Genève, septembre 2010, 2011

[https://plone2.unige.ch/aref20 I 0/symposiums-longs/coordinateursen-b/tourisme-et-

apprentissages/Les\%20professionnels\%20du\%20tourisme.pdf/view]. Jean-Didier URBAIN, Sur la plage. Mœurs et coutumes balnéaires (XIXXXe siècles), Payot, 1994.

Jean-Didier URBAIN, L'Idiot du voyage. Histoires de touristes, Payot, 2002.

John URrY, The Tourist Gaze, Sage, $2^{\circ}$ éd., 2002.

Etienne WENGER, La Théorie des communautés de pratique.

Apprentissage, sens et identité, Presses de l'université Laval, 2005. 